\title{
Current Insights into Immunotherapy Approaches for Food Allergy
}

Jessica D Macdougall (iD)

A Wesley Burks'

Edwin $\mathrm{H} \mathrm{Kim}^{2}$

'Department of Pediatrics, University of North Carolina School of Medicine, Chapel Hill, NC, USA; ${ }^{2}$ Department of Medicine, University of North Carolina School of Medicine, Chapel Hill, NC, USA
Correspondence: Edwin H Kim

Department of Medicine, University of North Carolina School of Medicine, 3004

Mary Ellen Jones Building, I 6 Manning

Dr, CB 8035, Chapel Hill, NC 27599, USA

$\mathrm{Tel}+\mid 9195373193$

Fax + I 9199625478

Email edwinkim@email.unc.edu
This article was published in the following Dove Press journal:

ImmunoTargets and Therapy

\begin{abstract}
In the last decade, there has been increasing research dedicated to food immunotherapy to induce clinical desensitization and provide protection by increasing clinical reaction thresholds. Results from recent food immunotherapy studies with differing routes of administration (oral, sublingual, and epicutaneous) suggest that food immunotherapy can induce clinical desensitization with varying levels of safety, however lasting tolerance has not been demonstrated. Furthermore, treatment side effects and dosing logistics may make the therapies difficult for some supporting the need for alternative treatment approaches. Peptide immunotherapy and DNA vaccine approaches should in theory allow for safer administration by decreasing allergenicity but proof of their clinical efficacy and immunogenicity remains to be proven. Biologic agents may allow for increased safety and rapid up-dosing of immunotherapy with the added benefit of treating multiple allergens simultaneously.
\end{abstract}

Keywords: food allergy, oral immunotherapy, sublingual immunotherapy, epicutaneous immunotherapy, omalizumab, dupilumab

\section{Introduction}

Food allergies impact approximately $11 \%$ of adults and $8 \%$ of children in the United States resulting in significant impairment in quality of life (QOL) for those affected and their families, as well as concern for life-threatening anaphylactic reactions prompting emergency room visits and hospitalizations. ${ }^{1,2}$ The most common parent-reported food allergies in children are to peanut $(2.2 \%)$, milk $(1.9 \%)$, shellfish $(1.3 \%)$, tree nut $(1.2 \%)$, and egg $(0.9 \%){ }^{2}$ Furthermore, multiple food allergies were reported by $40 \%$ of parents. ${ }^{2}$ Food allergy continues to be of concern due to the above-mentioned and the fact that the prevalence appears to be increasing prompting the need for further research into prevention and treatment. ${ }^{2}$

The standard of care for food allergy is avoidance of allergens and the use of epinephrine and antihistamines for treatment of allergic reactions. However, over the past decade, there has been increasing research dedicated to potential immunotherapy options for food allergy that would result in desensitization and a decreased frequency of allergic reactions after accidental ingestions. This review article will discuss current immunotherapy options for food allergy with a look toward future directions that may improve the safety and efficacy of food allergy treatment such as peptide immunotherapy, DNA plasmid vaccines, and biologic agents (as both monotherapy and as adjunct therapy to oral immunotherapy). 


\section{Present}

Food immunotherapy involves gradual administration of increasing doses or fixed doses of the specific allergen daily with the aim to increase the clinical reaction threshold. Food allergy research groups have studied several different ways to administer immunotherapy, including epicutaneous (EPIT), sublingual (SLIT), and oral (OIT). While immunotherapy to a number of allergenic foods have been reported, the majority of recent research studies have been specifically directed toward peanut immunotherapy given the number of people affected and the propensity for anaphylactic reactions.

\section{Oral Immunotherapy (OIT)}

OIT is the ingestion of food allergen protein into the GI tract to induce clinical desensitization. While OIT has been reported for many different food allergies including egg, milk and wheat, recent controlled clinical trials have been primarily focused on the treatment of peanut allergies. Studies have been conducted to evaluate for efficacy, safety, appropriate dosing, and immune modulation.

In one of the most comprehensive systematic reviews to date, Nurmatov, et al assessed 21 studies of food OIT across peanut, milk, and egg, as well as two Italian studies expanding therapy to apple, fish, peach, orange, corn, bean and lettuce, ${ }^{3}$ Over the 573 participants studied, a strong desensitization effect was demonstrated with a risk ratio of 0.14 (95\% CI 0.08, 0.24). This treatment effect was seen for cow's milk, egg and peanut OIT studies. However, a subgroup analysis based on age suggested that the efficacy was limited to participants' age 18 years and younger. Although complicated by varying reporting conventions between the studies, there was an increased risk of experiencing both systemic as well as local reactions with OIT when compared to controls. Although limited to one study, it was noteworthy that no improvement in QOL was found with OIT when compared to controls.

The 2019 PACE systematic review and meta-analysis more specifically reviewed the safety and efficacy of peanut OIT through December 2018 and included 12 randomized controlled trials encompassing a combined 1041 enrolled participants. ${ }^{4}$ Trial design varied across the studies with starting doses ranging from 0.1 to $2 \mathrm{mg}$ peanut protein, maintenance doses ranging from 125 to $4000 \mathrm{mg}$, and treatment duration ranging from 6 months to 5 years. Efficacy in protecting against allergic reactions and anaphylaxis as measured by in-clinic graded oral food challenges (OFCs) was significantly more likely for those on peanut OIT than those not on peanut OIT (RR 12.42 [95\% CI 6.82-22.61]). However, this was counterbalanced by markers of increased participant risk. Specifically, the authors reported that peanut OIT increased the risk of anaphylaxis (RR 3.12 95\% CI [1.76-5.55]), risk of epinephrine use (RR 2.21 95\% CI [1.27-3.83]), and risk of serious adverse events (SAEs) (RR 1.34 95\% CI [1.12-1.60]) compared with no peanut OIT. There was high evidence of increased risk of gastrointestinal and mucocutaneous reactions with peanut OIT. Regarding gastrointestinal reactions, three participants across five trials $(n=719)$ were diagnosed with eosinophilic esophagitis after peanut OIT. As seen in the Nurmatov review, despite the positive efficacy of peanut OIT, improvement in QOL using standardized tools was not observed for participants across any measures in the four studies reporting these results.

While the majority of studies have demonstrated shortterm efficacy with food OIT, the POISED study, a Phase 2 $\mathrm{RCT}$, focused on the long-term sustained unresponsiveness (SU) effect of peanut OIT to better understand the durability of desensitization after discontinuing therapy and the potential for tolerance. ${ }^{5}$ In this novel study design, a 2-year buildup and maintenance period with $4000 \mathrm{mg}$ of peanut OIT versus placebo was followed by either discontinuation of peanut OIT (Peanut-0) or continued treatment with a lower maintenance dose of $300 \mathrm{mg}$ (Peanut-300) versus placebo. In a change from the typical 1-month avoidance period, assessments of SU were done by double-blind, placebo-controlled food challenges (DBPCFCs) at 3-month intervals up to 1 year. The primary outcome of the study was met with $35 \%$ of Peanut- 0 compared to $4 \%$ of placebo participants completing a $4000 \mathrm{mg}$ peanut protein DBPCFC without symptoms 3 months after discontinuing OIT. While there was not a significant difference between Peanut- 0 and placebo participants 6,9 , and 12 months after discontinuing OIT, there was a significant difference in Peanut- 0 and Peanut300 participants at these time points with $13 \%$ of Peanut- 0 versus $37 \%$ of Peanut-300 participants completing the $4000 \mathrm{mg}$ DBPCFC 12 months after OIT discontinuation.

To date, it is unclear what OIT maintenance dose is most effective at maintaining clinical desensitization with minimal side effects. The data in the POISED study suggested that OIT is able to desensitize as seen in previous studies; however, discontinuation or even reduction of dose to $300 \mathrm{mg}$ peanut protein daily could result in an increased likelihood of regaining clinical reactivity to large 
doses of peanut. The results suggest with the strongest data to date that the desensitization effect is transient for most participants and likely requires regular and possibly high dose exposure to peanut to be maintained. ${ }^{5}$

While an in-depth review of biomarkers associated with OIT is beyond the scope of this review, the POISED study provided some additional insights into our understanding of the immune mechanisms of OIT. In particular, lower baseline peanut-specific IgE and Ara h 2 IgE was predictive of treatment success in both the Peanut- 0 and Peanut-300 groups. Lower basophil activation and higher peanut-specific $\operatorname{IgG} 4 / \operatorname{IgE}$ ratio were also associated with treatment success in the Peanut-0 group. Interestingly, the study found that a higher ratio of Ara h $2 \mathrm{IgE}$ to peanut-specific IgE was associated with treatment failure. Somewhat surprisingly, no association was seen with age, baseline peanut reaction threshold and peanut skin prick testing. ${ }^{5}$

\section{Epicutaneous Immunotherapy (EPIT)}

EPIT involves placing an adhesive dermal patch containing food protein onto the skin with the goal of inducing desensitization through a passive transfer of solubilized protein through the skin. The low dose exposure and nonoral administration route have been thought to provide a level of improved safety with the treatment when compared to OIT. Randomized controlled studies of EPIT have focused on peanut allergy using the proprietary Viaskin ${ }^{\circledR}$ patch system from DBV Technologies. A phase 2 multicenter study of 75 DBPCFC confirmed peanut-allergic participants conducted by the Consortium for Food Allergy Research (CoFAR) was designed to compare two peanut patches containing differing peanut protein amounts (100 mcg and $250 \mathrm{mcg}$ ) with placebo for 12 months of treatment. ${ }^{6}$ The study demonstrated that those randomized to either peanut-containing patch were statistically more likely than placebo to be responders as defined by either completing the end of treatment DBPCFC without dose-limiting symptoms or by increasing their DBPCFC reaction threshold by at least 10 -fold from baseline through the end of treatment. Specifically, $45.8 \%$ of the $100 \mathrm{mcg}$ peanut patch group and $48 \%$ in the 250 mcg peanut patch group were considered responders. With successfully consumed dose (SCD) defined as the cumulative amount of peanut protein ingested during DBPCFC without symptoms, the change in median SCD for the $100 \mathrm{mcg}$ and $250 \mathrm{mcg}$ groups after treatment was $43 \mathrm{mg}$ and $130 \mathrm{mg}$ of peanut protein, respectively. The clinical results were supported by evidence of immune modulation including increased peanut-specific IgG4 levels and peanut-specific IgG4/IgE ratios when compared to the placebo group. Importantly from this study, EPIT (whether low or high dose) was shown to be safe with the majority of reactions being mild and localized to the patch site.

\section{Sublingual Immunotherapy (SLIT)}

Whereas EPIT involves placing a patch on the skin, SLIT involves placing small amounts of the allergen via drops or tablets under the tongue to induce desensitization. Like EPIT, SLIT has been predicted to be well tolerated with fewer dose-limiting side effects given its route of administration and the small amount of allergen per dose. Prior studies demonstrated safety and efficacy in hazelnut ${ }^{7,8}$ and peach allergy, ${ }^{9}$ while recent studies have primarily focused on peanut allergy. Following up the positive results of our group's initial double-blind study of peanut SLIT, ${ }^{10}$ participants were continued in an unblinded, single arm, extension study receiving maintenance doses of peanut SLIT at $2 \mathrm{mg} /$ day peanut protein for up to a total of five years. ${ }^{11}$ With extended therapy, SLIT remained well tolerated with only $4.8 \%$ of doses associated with symptoms affecting $94 \%$ of participants. The majority of these symptoms were reported as mild and self-resolving with transient oropharyngeal itching being the most common. Other less common symptoms included lip swelling, rhinorrhea, sneeze, wheezing, cough, and mild gastrointestinal distress. Importantly, eosinophilic esophagitis (EoE) was not reported in this study and to date, while there have been reports of EoE with environmental allergen SLIT, ${ }^{12,13}$ there have been no reports from food allergen SLIT. In addition to its reassuring safety profile, there was also excellent compliance with $95.5 \%$ of doses successfully administered supporting the simple administration of the sublingual approach.

The 48 participants in our peanut SLIT extension study were assessed for efficacy with a $5000 \mathrm{mg}$ peanut protein DBPCFC to assess for desensitization after completion of SLIT dosing, then were instructed to stop treatment and avoid peanuts for up to two to four weeks to assess for SU. ${ }^{11}$ Twelve participants completed the desensitization DBPCFC without symptoms and discontinued SLIT. Of those twelve, 10 participants completed the subsequent DBPCFC without clinical symptoms demonstrating SU. As reported with OIT and EPIT, there was an immune modulatory effect with significantly decreased peanut- 
specific basophil activation, peanut skin testing, and peanut-specific immunoglobulins from baseline to end of treatment. It was also noted that two-thirds of participants in this study were able to tolerate at least $750 \mathrm{mg}$ peanut protein with a median SCD of $1750 \mathrm{mg}$ peanut protein for the cohort. This suggested a clinically significant buffer from accidental ingestions of peanut as increasing the reaction threshold to even $300 \mathrm{mg}$ peanut protein has been estimated to provide up to a $95 \%$ reduction of risk for peanut-allergic reactions after ingestion common packaged goods. ${ }^{14}$ Further studies are needed to assess any effects on QOL for patients and their families after SLIT therapy.

Of interest to many practitioners is a comparison of the different immunotherapy modalities; however, direct comparisons have been lacking. One novel, randomized, single-center study by Narisety, et al directly compared OIT with SLIT for peanut allergy. ${ }^{15}$ Participants were initially treated with OIT or SLIT with those not achieving desensitization then adding the alternative treatment as a combined therapy. Although OIT appeared to increase the reaction threshold greater than SLIT, 141-fold versus 22-fold, respectively, SLIT was still able to significantly increase the reaction threshold from baseline with the median SCD increasing from baseline of $21 \mathrm{mg}$ peanut protein to $496 \mathrm{mg}$ peanut protein after 12 months of treatment. In addition, there were less symptoms related to SLIT doses (9\% of doses affecting $90 \%$ of participants) in comparison to OIT doses ( $43 \%$ of doses affecting $100 \%$ of participants) and more oropharyngeal, respiratory and gastrointestinal symptoms with OIT when compared to SLIT.

\section{Future Directions}

\section{Peptide Immunotherapy}

OIT has demonstrated improved clinical desensitization to an allergen and peanut OIT has recently been approved by the FDA for peanut allergy; however, being administered as an intact protein runs the risk of binding and crosslinking IgE leading to increased allergic reactions. This was shown by the $98.7 \%$ of active drug participants in the PALISADE Phase 3 peanut OIT trial reporting adverse events during the intervention period. ${ }^{16}$ Peptides, on the other hand, are thought to be less likely to activate mast cells or basophils, while maintaining the immunogenic ability to be recognized by T cells. ${ }^{17}$ While the concept of using non-intact proteins for immunotherapy has been considered in the past, the potential safety benefits of the approach have taken on a renewed level of importance as OIT becomes poised to be offered broadly.

In a small Phase I study in Australia of 66 participants who underwent intradermal injection with a product consisting of several synthetic peptides derived from peanut allergens (PVX108; Aravax, Melbourne, Australia), the decreased allergenicity of peptide immunotherapy was demonstrated by a lack of basophil activation to the immunotherapy. ${ }^{18}$ During the study, there were no serious adverse events and the AEs that did occur were mostly self-resolving injection site reactions. Although the study was limited by small sample size, the results indicated that peptide immunotherapy may prove advantageous in regards to reducing adverse reactions that may otherwise preclude use by an allergic individual. As Phase I data pertained to the safety profile, future studies would be needed to ascertain if peptide immunotherapy is able to induce clinical desensitization and if so, the degree and duration of this desensitization effect.

\section{DNA Vaccines}

Another emerging therapy with a goal of maintaining immunogenicity while limiting allergenicity is the development of a single multivalent peanut (Ara h1, h2, h3) lysosomal associated membrane protein DNA plasmid vaccine that is currently undergoing Phase 1 trials for safety. ${ }^{19,20}$ A plasmid DNA vaccine encoding allergens is hypothesized to induce T-helper 1 and regulatory $\mathrm{T}$ cell responses without activation of mast cells. ${ }^{21}$ This has the potential to counteract allergic T-helper 2-biased reactions, including those experienced with food allergy.

\section{Monoclonal Antibody Therapies}

Concurrent with the advances in EPIT, OIT and SLIT, there has been increasing research exploring biologic agents, e.g. omalizumab and dupilumab, with the hope of improved safety, stronger benefit, and perhaps most importantly allergen non-specificity. Biologics have been studied as both adjunctive therapy to OIT as well as in monotherapy for food allergies.

Omalizumab is a monoclonal anti-IgE antibody with FDA approval for the treatment of allergic asthma and chronic idiopathic urticaria. As an investigational product, it has been used in food allergy studies to facilitate rapid desensitization with OIT by decreasing adverse reactions, especially during up-dosing phases. A randomized, double-blind, placebo-controlled, multi-center trial studied the 
effects of omalizumab versus placebo as an adjunct to peanut OIT in 37 peanut-allergic participants with results indicating that omalizumab allowed for rapid up-dosing of peanut OIT. ${ }^{22}$ In comparison to the PALISADE trial where it took participants seven months to escalate to an OIT dose of $300 \mathrm{mg}$ of peanut protein, ${ }^{16}$ of those in the omalizumab group $85 \%(n=23$ of 27$)$ escalated to an OIT dose of $250 \mathrm{mg}$ peanut protein on the first day. In comparison, those in the placebo group reached a median OIT dose of $22.5 \mathrm{mg}$ peanut protein on the first day. Dose escalation progressed more quickly when compared to other peanut OIT trials with $72 \%$ of the omalizumab group escalating to $2000 \mathrm{mg}$ peanut protein by week 20 of the intervention. Of participants on omalizumab, $85 \%$ (23 of 27) remained able to tolerate the $2000 \mathrm{mg}$ peanut OIT dose six weeks following omalizumab discontinuation suggesting that desensitization to the peanut OIT was maintained in comparison to $12.5 \%$ ( 1 of 8 ) in the placebo group. There was a lower percentage of OIT dosing reactions in the omalizumab group versus placebo ( $7.8 \%$ vs $16.8 \%$, respectively) but this was not statistically significant. There were no significant reactions attributable to omalizumab indicating that this may be a safe option to enable patients to reach maintenance doses of OIT more quickly.

While the first study demonstrated sustained desensitization following discontinuation, a more recent study investigating 23 peanut-allergic adolescents who received omalizumab for eight weeks prior to initiating OIT and then continued omalizumab as an adjunct to peanut OIT until reaching the $2800 \mathrm{mg}$ peanut protein maintenance dose did not demonstrate a sustained response in a majority of participants. ${ }^{23}$ As in the MacGinnitie study, a strong omalizumab treatment effect was seen with all participants able to reach the $2800 \mathrm{mg}$ peanut OIT maintenance dose while receiving omalizumab in a median time of 10 weeks. However, only $48 \%$ of participants were able to continue tolerating the $2800 \mathrm{mg}$ peanut OIT dose 12 weeks after discontinuing omalizumab. Interestingly, in looking at biomarker data, the group tolerating peanut OIT following omalizumab discontinuation had lower median baseline $\operatorname{IgE}$ to peanut and components Ara h 1-3, as well as significantly lower baseline IgG4 to Ara h 2 and 6 compared to the intolerant group. IgG4 levels to peanut increased during the maintenance phase; however, levels for control allergen did not change suggesting that the difference in biomarkers was induced by peanut OIT rather than omalizumab. Basophil reactivity was decreased during omalizumab pre-treatment but began to increase as omalizumab doses were lowered or discontinued indicating that there is not a sustained response once the individual was no longer receiving omalizumab. Importantly, preliminary data from long-term follow-up of participants on peanut OIT demonstrated that allergic reactions were less frequent and when reactions occurred it was during times of worse compliance which may hint at the need for continued patient education regarding the goals and limitations of OIT.

The OUtMATCH study is a new multi-center, randomized controlled trial that intends to further explore the role of omalizumab as a monotherapy and an adjunct therapy to OIT for individuals with multiple food allergies (peanut plus at least two other foods). ${ }^{24}$ The study will be performed in three stages: Stage 1 is four months of omalizumab monotherapy versus placebo, Stage 2 is fourteen months of extended therapy omalizumab versus omalizumab-facilitated multi-food OIT, and Stage 3 is the transition to real-world food forms to maintain the desensitized state while expanding the diet. Of particular interest with this study will be the potential for omalizumab to treat multiple food allergies simultaneously.

A second biologic agent of interest for treatment of food allergy is dupilumab, a human monoclonal antibody that acts as a dual inhibitor of IL-4 and IL-13 signaling. ${ }^{25}$ As IL-4 and IL-13 are cytokines that play an important role in $\mathrm{T}_{\mathrm{H}} 2$ allergic inflammation, there is hope that inhibition of these cytokines may reduce or even eliminate allergic reactions to allergens. Dupilumab is currently approved for the treatment of atopic dermatitis, asthma, and chronic rhinosinusitis with nasal polyposis; however, there have been case reports of decreased reactions to food allergens as well. There are several ongoing clinical trials to evaluate dupilumab as either a monotherapy or as an adjunct to OIT for peanut allergy.

In one current phase 2 trial, participants receive either dupilumab or placebo in conjunction with peanut OIT to determine whether dupilumab serves to improve desensitization by increasing the proportion of participants that are able to complete a $2044 \mathrm{mg}$ peanut protein DBPCFC without dose-limiting symptoms after dose escalation. It will secondarily assess whether desensitization is maintained compared to placebo, what the immune modulatory effects are, and its safety and tolerability profile. ${ }^{26}$ In parallel, dupilumab is being studied as monotherapy in peanut-allergic children with the primary outcome assessing the proportion of patients treated with dupilumab who subsequently complete a $444 \mathrm{mg}$ peanut protein $\mathrm{DBPCFC}$ 
without dose-limiting symptoms after 24 weeks of therapy. ${ }^{27}$

Beyond peanut allergy, a phase 2 randomized clinical trial to evaluate dupilumab as an adjunct to milk OIT has been developed with the hope of improving safety and rates of desensitization. ${ }^{28}$ Those in the treatment arm will receive dupilumab prior to and during up-dosing of milk OIT followed by eight weeks of milk OIT without dupilumab to evaluate the proportion of participants that are able to tolerate at least $2040 \mathrm{mg}$ cumulative cow's milk protein at week eighteen.

\section{Discussion}

Food allergy can result in life-threatening anaphylactic reactions and significant impairment in quality of life that has prompted research efforts to develop therapies that will lead to clinical desensitization and protection against accidental ingestions of food allergen colloquially referred to as bite-proof protection. When discussing EPIT, SLIT and OIT, efficacy has been typically measured by reaction thresholds during DBPCFC. However, the level of desensitization that provides clinically meaningful protection against accidental ingestions is a critical concept that has remained poorly studied due to the inherently infrequent and unpredictable nature of accidental ingestions. Food allergen ingestion modeling by Baumert, et al at the University of Nebraska Food Allergy Research and Resource Laboratory estimated that increasing a patient's reaction threshold to $300 \mathrm{mg}$ of peanut protein could provide a greater than $95 \%$ risk reduction from peanutallergic reactions after ingestion of common packaged foods. ${ }^{14}$ While this data would support a level of efficacy with all three modalities of immunotherapy, additional factors that need to be considered include desire for a buffer against larger exposures, ease of treatment administration, cost, and safety of the treatment. What these factors make clear is that there is no single perfect treatment for peanut allergy at this time. Furthermore, the best approach for peanut may not be the best approach for other foods. Each modality has its own specific strengths and weaknesses that have to be aligned with the patient and provider's goals for therapy. With EPIT, SLIT and OIT likely requiring long-term if not life-long treatment, even small inconveniences and risks can become significant over time highlighting the need for continued research into peanut allergy treatments.

Peptide immunotherapy is one option that could be beneficial as it would be expected to have similar immune modulatory effects on T cells as with standard food immunotherapy but with a diminished risk of triggering immediate effector cells such as mast cells and basophils. DNA plasmid vaccines have the similar advantage of bypassing immediate effector cells with the added potential of modifying and reducing the allergic response. While the safety of these treatments is anticipated, larger phase 2 studies are needed to demonstrate the degree of desensitization possible with these approaches.

There is current data to suggest a near-term future role for biologic agents, specifically omalizumab and dupilumab, in the treatment of food allergy. It has been shown that omalizumab is able to facilitate rapid dose escalation of peanut OIT, as well as improve the safety of OIT. The OUtMATCH clinical trial is expected to provide additional data on the use of omalizumab as an adjunctive therapy to OIT, but more importantly, the trial seeks to show the efficacy of omalizumab across multiple food allergens simultaneously potentially providing a tremendous advance in the treatment of food allergy. Given its $\mathrm{T}_{\mathrm{H}} 2$ suppressive mechanism of action, dupilumab may also prove effective both as an adjunctive therapy and as monotherapy. With their current availability for the treatment of other atopic diseases, these biologics could reach patients faster than novel therapeutics in development and are a source of great anticipation for the near horizon; however, these benefits will need to be weighed against the likely high cost of many of these treatments that may make them cost-prohibitive for many allergic patients.

\section{Conclusion}

While clinical trials have shown clinical desensitization and immune modulation with EPIT, SLIT and OIT for food allergy, there are still many questions regarding safety and tolerability as these therapies have not been shown to elicit long-term tolerance. The development of alternative treatment approaches for food allergy such as peptide immunotherapy, DNA vaccines and biologics hope to improve on the safety of food allergy treatment while potentially providing additional benefits such as rapid desensitization, longerlasting efficacy, and treatment across multiple allergens simultaneously. These advances give us a glimpse of the future of food allergy treatment and continue to build on the hope for the eventual cure for all food allergies.

\section{Abbreviations}

AE, adverse events; CoFAR, Consortium for Food Allergy Research; DBPCFC, double-blind placebo-controlled food 
challenge; EPIT, epicutaneous immunotherapy; IgE, immunoglobulin E; IgG4, immunoglobulin G4; OFC, oral food challenge; OIT, oral immunotherapy; QOL, quality of life; RCT, randomized controlled trial; SCD, successfully consumed dose; SLIT, sublingual immunotherapy; SU, sustained unresponsiveness.

\section{Disclosure}

JD Macdougall reports no conflicts of interest in this work. AW Burks reports being a minority stock holder in Allertein, Mastcell pharmaceuticals; scientific advisory board membership with Aimmune Therapeutics, Consortia TX Inc, Prota therapeutics; consultancy for Astella Pharma Global Development, DBV technologies, N-fold LLC, kaleo, Ukko Inc; royalties with UpToDate; and receives grant support to his institution from the NIH/ NIAID, NIH/NCCIH, FARE and the Wallace Research Foundation. In addition, AW Burks has patents US $7,879,977$, US 6,835,824, US 6,486,311, US 6,441,142, US 5,973,121, and US 5,558,869 issued. EH Kim reports clinical medical advisory board membership with DBV Technologies; consultancy with Aimmune Therapeutics, DBV Technologies, AllerGenis, Allakos, Kenota Health, Ukko, and Vibrant America; and receives grant support to his institution from the National Institute of Allergy and Infectious Diseases (NIH/NIAID), National Center for Complementary and Integrative Health (NIH/NCCIH), FARE and the Wallace Research Foundation. The authors report no other conflicts of interest in this work.

\section{References}

1. Gupta RS, Warren CM, Smith BM, et al. Prevalence and severity of food allergies among US adults. JAMA Netw Open. 2019;2(1): e185630. doi:10.1001/jamanetworkopen.2018.5630

2. Gupta RS, Warren CM, Smith BM, et al. The public health impact of parent-reported childhood food allergies in the United States. Pediatrics. 2018;142(6):e20181235. doi:10.1542/peds.2018-1235

3. Nurmatov U, Dhami S, Arasi S, et al. Allergen immunotherapy for IgE-mediated food allergy: a systematic review and meta-analysis. Allergy. 2017;72(8):1133-1147. doi:10.1111/all.13124

4. Chu DK, Wood RA, French S, et al. Oral immunotherapy for peanut allergy (PACE): a systematic review and meta-analysis of efficacy and safety. Lancet. 2019;393(10187):2222-2232. doi:10.1016/S01406736(19)30420-9

5. Chinthrajah RS, Purington N, Andorf S, et al. Sustained outcomes in oral immunotherapy for peanut allergy (POISED study): a large, randomised, double-blind, placebo-controlled, phase 2 study. Lancet. 2019;394(10207):1437-1449. doi:10.1016/S0140-6736(19)31793-3

6. Jones SM, Sicherer SH, Burks AW, et al. Epicutaneous immunotherapy for the treatment of peanut allergy in children and young adults. J Allergy Clin Immunol. 2017;139(4):1242-1252 e1249. doi:10.1016/j. jaci.2016.08.017
7. Enrique E, Pineda F, Malek T, et al. Sublingual immunotherapy for hazelnut food allergy: a randomized, double-blind, placebo-controlled study with a standardized hazelnut extract. J Allergy Clin Immunol. 2005;116(5):1073-1079. doi:10.1016/j.jaci.2005.08.027

8. Enrique E, Malek T, Pineda F, et al. Sublingual immunotherapy for hazelnut food allergy: a follow-up study. Ann Allergy Asthma Immunol. 2008;100(3):283-284. doi:10.1016/S1081-1206(10)60456-5

9. Fernandez-Rivas M, Garrido Fernandez S, Nadal JA, et al. Randomized double-blind, placebo-controlled trial of sublingual immunotherapy with a Pru p 3 quantified peach extract. Allergy. 2009;64(6):876-883. doi:10.1111/j.1398-9995.2008.01921.x

10. Kim EH, Bird JA, Kulis M, et al. Sublingual immunotherapy for peanut allergy: clinical and immunologic evidence of desensitization. J Allergy Clin Immunol. 2011;127(3):640-646 e641. doi:10.1016/j. jaci.2010.12.1083

11. Kim EH, Yang L, Ye P, et al. Long-term sublingual immunotherapy for peanut allergy in children: clinical and immunologic evidence of desensitization. J Allergy Clin Immunol. 2019;144(5):1320-1326.e1. doi:10.1016/j.jaci.2019.07.030

12. Bene J, Ley D, Roboubi R, Gottrand F, Gautier S. Eosinophilic esophagitis after desensitization to dust mites with sublingual immunotherapy. Ann Allergy Asthma Immunol. 2016;116 (6):583-584. doi:10.1016/j.anai.2016.03.017

13. Kawashima K, Ishihara S, Masuhara M, et al. Development of eosinophilic esophagitis following sublingual immunotherapy with cedar pollen extract: a case report. Allergol Int. 2018;67 (4):515-517. doi:10.1016/j.alit.2018.03.003

14. Baumert JL, Taylor SL, Koppelman SJ. Quantitative assessment of the safety benefits associated with increasing clinical peanut thresholds through immunotherapy. J Allergy Clin Immunol Pract. 2018;6 (2):457-465 e454. doi:10.1016/j.jaip.2017.05.006

15. Narisety SD, Frischmeyer-Guerrerio PA, Keet CA, et al. A randomized, double-blind, placebo-controlled pilot study of sublingual versus oral immunotherapy for the treatment of peanut allergy. J Allergy Clin Immunol. 2015;135(5):1275-1282. doi:10.1016/j.jaci.2014.11.005

16. Investigators PGoC, Vickery BP, Vereda A, et al. AR101 oral immunotherapy for peanut allergy. $N$ Engl J Med. 2018;379(21):1991-2001.

17. El-Qutob D, Reche P, Subiza JL, Fernandez-Caldas E. Peptide-based allergen specific immunotherapy for the treatment of allergic disorders. Recent Pat Inflamm Allergy Drug Discov. 2015;9 (1):16-22. doi:10.2174/1872213X09666150302105555

18. Prickett SR, Hickey PL, Bingham J, et al. Safety and tolerability of a novel peptide-based immunotherapy for peanut allergy. J Allergy Clin Immunol. 2019;143(2):AB431. doi:10.1016/j.jaci.2018.12.975

19. A study to evaluate safety, tolerability and immune response in adolescents allergic to peanut after receiving intradermal administration of ASP0892 (ARA-LAMP-vax), a single multivalent peanut (Ara h1, h2, h3) lysosomal associated membrane protein DNA plasmid vaccine. Available from: https://ClinicalTrials.gov/show/ NCT03755713. Accessed January 13, 2021.

20. A study to evaluate safety, tolerability and immune response in adults allergic to peanut after receiving intradermal or intramuscular administration of ASP0892 (ARA-LAMP-vax), a single multivalent peanut (Ara h1, h2, h3) lysosomal associated membrane protein DNA plasmid vaccine. Available from: https://ClinicalTrials.gov/show/ NCT02851277. Accessed January 13, 2021.

21. Scheiblhofer $\mathrm{S}$, Thalhamer J, Weiss R. DNA and mRNA vaccination against allergies. Pediatr Allergy Immunol. 2018;29(7):679-688. doi:10.1111/pai.12964

22. MacGinnitie AJ, Rachid R, Gragg H, et al. Omalizumab facilitates rapid oral desensitization for peanut allergy. J Allergy Clin Immunol. 2017;139(3):873-881 e878. doi:10.1016/j.jaci.2016.08.010

23. Brandstrom J, Vetander M, Sundqvist AC, et al. Individually dosed omalizumab facilitates peanut oral immunotherapy in peanut allergic adolescents. Clin Exp Allergy. 2019;49(10):1328-1341. doi:10.1111/ cea. 13469 
24. Omalizumab as monotherapy and as adjunct therapy to multi-allergen OIT in food allergic participants. Available from: https:// ClinicalTrials.gov/show/NCT03881696. Accessed January 13, 2021.

25. Sastre J, Davila I. Dupilumab: a new paradigm for the treatment of allergic diseases. J Investig Allergol Clin Immunol. 2018;28 (3):139-150. doi:10.18176/jiaci.0254

26. Study in pediatric subjects with peanut allergy to evaluate efficacy and safety of dupilumab as adjunct to AR101 (peanut oral immunotherapy). Available from: https://ClinicalTrials.gov/show/ NCT03682770. Accessed January 13, 2021.
27. Study to Evaluate Dupilumab Monotherapy in Pediatric Patients With Peanut Allergy. Available from: https://ClinicalTrials.gov/show/ NCT03793608. Accessed January 13, 2021.

28. Dupilumab and Milk OIT for the Treatment of Cow's Milk Allergy. Available from: https://ClinicalTrials.gov/show/NCT04148352. Accessed January 13, 2021.

\section{Publish your work in this journal}

ImmunoTargets and Therapy is an international, peer-reviewed open access journal focusing on the immunological basis of diseases, potential targets for immune based therapy and treatment protocols employed to improve patient management. Basic immunology and physiology of the immune system in health, and disease will be also covered. In addition, the journal will focus on the impact of management programs and new therapeutic agents and protocols on patient perspectives such as quality of life, adherence and satisfaction. The manuscript management system is completely online and includes a very quick and fair peer-review system, which is all easy to use. Visit http://www.dovepress.com/testimonials.php to read real quotes from published authors. 